\section{P2-286 SYSTEMATIC REVIEW WITH META-ANALYSIS OF POPULATION-BASED VS SCHOOL-BASED STUDIES OF OBESITY PREVALENCE AMONG ADOLESCENTS FROM BRAZIL}

doi:10.1136/jech.2011.142976k.19

${ }^{1}$ B Soldateli, ${ }^{1,2}$ S Fuchs, ${ }^{* 1}{ }^{1}$ S Schmidt. ${ }^{1}$ Postgraduate Studies Program in Epidemiology, Universidade Federal do Rio Grande do Sul, Porto Alegre, Rio Grande do Sul, Brazil; ${ }^{2}$ Hospital de Clínicas de Porto Alegre, Porto Alegre, Rio Grande do Sul, Brazil

Introduction In Brazil, obesity prevalence in adolescents has been investigated in different regions and periods of time. Studies of school-based samples have low cost and are easily conducted, being widely used. Otherwise, population-based studies require greatest investments, more time and researches, besides high complexity to minimise bias.

Objective To undertake meta-analysis of observational studies conducted in Brazilian adolescents to calculate pooled estimates for obesity prevalence for school-based vs population-based samplings.

Methods Data sources included Medline, Embase, Bireme, and CAPES-Thesis database searching for articles and grey literature about obesity prevalence in adolescents aged 10-19 years, from studies conducted in Brazil from 1980 to 2010. Studies were systematically reviewed, with no language restriction, and independently extracted by two investigators using a standardised protocol. Obesity was defined by body mass index (BMI) $\geq 95$ Percentile, for sex and age. Data analysis was performed by Comprehensive Meta-Analysis $2.0 \circledR$, using random effect model.

Results Among 1939 articles reviewed, 138 met the inclusion criteria, 56 were excluded, and 25 were not retrieved after three attempts to contact authors. Among 57 studies included in metaanalysis 14 were population-based and 43 school-based samples. No significant heterogeneity was detected $(Q=8.25, p=0.001)$. Obesity prevalence in population-based studies was 5.5 (95\% CI 4.2 to 7.1 ) and in school-based studies it was 5.1 (95\% CI 4.3 to 6.0). Schoolbased studies showed higher inconsistency measure of results when compared with population-based samples studies $\left(\mathrm{I}^{2}=7.74\right.$ vs 0$)$.

Conclusion School-based studies as well population-based studies provide similar estimates of obesity prevalence, since they are rigorously conducted regarding methodological aspects.

\section{P2-287 SEROPREVALENCE AND INCIDENCE STUDY OF HIV IN TRANSGENDER IN ARGENTINA -2009}

doi:10.1136/jech.2011.142976k.20

J Sotelo, ${ }^{*}$ B Claudia. Ministry of Health of the Nation, Ciudad Autónoma de Buenos Aires, Argentina

Introduction To determine the incidence and seroprevalence of HIV in Transgender and its association with risk behaviour at a national level. We realised a survey study where 455 Trans people were polled about demographic, cultural and behavioural aspects in 12 Argentine provinces. They received pre-HIV test counselling and realised serology. For calculating the incidence was applied the STARHS algorithm - $\mathrm{I}=(\mathrm{n} / \mathrm{m}+\mathrm{n})) \times(365.25 / \mathrm{T}) \times 100$.

Results We analysed data from 441 surveyed: 152 positive cases $(\mathrm{P})$, 276 negative $(\mathrm{N})$ and 13 indeterminate (I); Prevalence was $34.47 \%$ (95\% CI 30.04 to 39.11 ). $63.41 \%$ of $\mathrm{N}$ and $73.68 \%$ of $\mathrm{P}$ had used silicone injections $(p=0.03)$. 316/441 were sexual workers $(S W), 119$ $(37.66 \%)$ were P $187 \mathrm{~N}$ and 10 I. 35,4\% of SW used condom with his last client. $54.01 \%$ of SW N and $64.71 \%$ of SW P used drugs with clients $(p=0064)$. Estimated incidence HIV was 11.14 per 100 person-years (95\% CI 4.98 to 20.99). The annual incidence was calculated using a window period of 170 days set for HIV-1 subtype B.

Conclusions The prevalence was similar to the reported in other Transgender populations (USA, Canada) and was greater in the subgroup of Sexual workers. Knowledge of other sexually transmitted diseases and use of silicone injections were significantly associated with the positive condition (HIV+). Vulnerability in sexual workers is associated with the increased consumption of alcohol, drugs, social discrimination and police violence.

\section{P2-288 TREND OF LUNG CANCER MORTALITY IN BRAZIL, 1980 TO 2007}

doi:10.1136/jech.2011.142976k.21

${ }^{1,2} \mathrm{M}$ C de Souza, ${ }^{*}$ A G G Vasconcelos, ${ }^{4} 0$ G Cruz. ${ }^{1}$ Instituto Nacional de Câncer (INCA), Rio de Janeiro, Rio de Janeiro, Brazil; ${ }^{2}$ Programa de Pós-Graduação em Epidemiologia em Saúde Pública, Escola Nacional de Saúde Pública Sérgio Arouca (ENSP), Fundação Oswaldo Cruz (Fiocruz), Rio de Janeiro, Rio de Janeiro, Brazil; ${ }^{3}$ Departamento de Métodos Quantitativos em Saúde, Escola Nacional de Saúde Pública Sérgio Arouca (ENSP), Fundação Oswaldo Cruz (Fiocruz), Rio de Janeiro, Rio de Janeiro, Brazil; ${ }^{4}$ Programa de Computação Científica (PROCC), Fundação Oswaldo Cruz (Fiocruz), Rio de Janeiro, Rio de Janeiro, Brazil

Objectives To describe the trends in mortality from lung cancer in Brazil and to identify the effects of age, period and birth cohort on these rates by sex.

Methods We conducted a time series study and calculated mortality rates (crude, specific and adjusted by age) from lung cancer, by sex between 1980 and 2007. To identify how age, period and birth cohort influence mortality rates from lung cancer APC models were adjusted.

Results The mortality rate from lung cancer is significantly higher among men compared the rates of women. The specific rates for men aged over 64 and women at all ages, are increasing. The adjusted rates had a greater increase among women. The effect of age indicates that the mortality risk increases rapidly from the early ages. The results of the birth cohort effect for men indicate a lower risk for those born after 1950 and an increased risk for women in all cohorts.

Conclusions The results of the younger generations indicate that current trends in mortality rates from lung cancer should be maintained for some years. The cohort effect observed among women born after 1925 suggests a mortality increase. The reduction in mortality among men under 65 , suggests that, the trend started among those born after 1950, will continue. These trends are connected to the tobacco control measures.

\section{P2-289 OBESITY AND WHOLE BLOOD SEROTONIN IN HEALTHY VOLUNTEERS}

doi:10.1136/jech.2011.142976k.22

S J Hodge, B P Bunting, J J Strain, B J Stewart-Knox. * University of Ulster, Coleraine, UK

The aim of this study was to explore associations between fasting whole blood serotonin levels and anthropometric measures in healthy male and female volunteers $(\mathrm{N}=68)$ aged between 20 and 66 years. A fasting $(20 \mathrm{ml})$ venous blood sample for whole blood serotonin was collected, after which body mass index and waist circumference (WC) were measured by DEXA (dual energy x-ray absorptiometry) scan. Data were analysed using the SPSS (Statistical Package for the Social Sciences v 17.0) software. Pearson 
(partial) correlations showed significant associations between whole blood serotonin and BMI $(r=-0.284, p=0.021)$ and waist circumference $(r=-0.325, p=0.008)$. The correlational nature of these analyses do not enable conclusions to be drawn as to cause and effect but suggest an interplay between obesity and lower whole blood serotonin levels.

Funding This analysis has been funded by the HRB (Ireland) Health Research Centre.

\section{P2-290 SOCIOECONOMIC STATUS AND MORTALITY AMONG PEOPLE WITH DIABETES MELLITUS IN LATVIA}

doi:10.1136/jech.2011.142976k.23

${ }^{1}$ I Strele, ${ }^{* 1,2} \mathrm{~L}$ Golosujeva, ${ }^{1,2} \mathrm{~S}$ Rozite, ${ }^{1} \mathrm{G}$ Brigis, ${ }^{3} \mathrm{~V}$ Pirags. ${ }^{1}$ Riga Stradins University, Riga, Latvia; ${ }^{2}$ Centre of Health Economics, Riga, Latvia; ${ }^{3}$ University of Latvia, Riga, Latvia

Introduction The chronic course of the disease and regular need for healthcare suggests that people with diabetes mellitus might be more affected by the socioeconomic inequalities in health. We assessed whether socioeconomic differences exist in mortality of diabetes patients in Latvia.

Methods The study population consisted of 4179 diabetes patients (1339 men and 2840 women) participating in the Latvian diabetes survey in 2000. All deaths $(n=1771)$ during a subsequent ten-year period and causes of death were identified through the Causes of Death Data Base. The Cox model was used to test associations (HR ( $95 \% \mathrm{CI}$ ) presented) between education, income and residence (rural/urban) and mortality, adjusted for age, duration of diabetes, glycaemia, and blood pressure.

Results The primary education, compared to the higher education, was associated with an increase in all-cause, cardiovascular mortality and death from diabetes in both men (1.45 (1.16-1.82); 1.45 (1.07-1.95); 2.33 (1.23-4.40), respectively) and women (1.91 (1.53-2.38); 1.72 (1.31-2.25); 2.37 (1.25-4.48), respectively). The lowest income tertile, compared to the highest, was associated with higher all-cause mortality in both men (1.42 (1.14-1.77)) and women (1.37 (1.14-1.65)), as well as with higher cardiovascular mortality in men (1.44 (1.06-1.94)). Rural residence was associated with lower cardiovascular mortality in men $(0.70(0.50-0.98))$. The strength of associations did not change substantially when smoking, alcohol consumption, or parameters of diabetes care were included in the model.

Conclusion Overall, diabetes patients with poorer education and lower income experienced higher mortality. These inequalities were not explained by behavioural factors or diabetes care.

\section{P2-291 STRUCTURAL AND FUNCTIONAL MEASURES OF SOCIAL SUPPORT, SOCIOECONOMIC POSITION AND MORTALITY. THE BRITISH WHITEHALL II STUDY}

doi:10.1136/jech.2011.142976k.24

${ }^{1} \mathrm{~S}$ Stringhini, ${ }^{2} \mathrm{~L}$ Berkman, ${ }^{1} \mathrm{~A}$ Dugravot, ${ }^{3} \mathrm{~J}$ E Ferrie, ${ }^{3} \mathrm{M}$ Marmot, ${ }^{3} \mathrm{M}$ Kivimaki, ${ }^{1,3}$ A Singh-Manoux. ${ }^{1}$ INSERM U1018, Paris, France, ${ }^{2}$ Harvard school of Public Health, Boston, USA; ${ }^{3}$ University College London, London, UK

Introduction Social relationships are associated with socioeconomic position (SEP) and with mortality, but it is unclear to what extent the socioeconomic gradient in mortality is attributable to differences in social relations between socioeconomic groups. In this study, we examine the contribution of different measures of social support to the socioeconomic gradient in all-cause and cause-specific mortality.
Methods Analyses are based on 9333 participants from the British Whitehall II study, aged 35-55 years at baseline (1985). SEP was civil service employment grade at baseline and social support was assessed 3 times over a follow-up for mortality of 24.4 years.

Results Measures of social support were socially patterned, but the pattern differed by gender. In men, social isolation and marital status were predictors of all-cause $(\mathrm{HR}=1.27,95 \%$ CI 1.07 to 1.52 for low network score and $\mathrm{HR}=1.77$; $95 \%$ CI 1.45 to 2.16 for not being married) and cardiovascular mortality $(\mathrm{HR}=1.85$; 95\% CI 1.36 to 2.51 for low network score and $\mathrm{HR}=2.69$; $95 \% \mathrm{CI}$ 1.95 to 3.71 for not being married). These associations were partially attenuated after adjustment for self-rated health. Men in the lowest SEP had an higher mortality risk than those in the highest $(\mathrm{HR}=1.59 ; 95 \% \mathrm{CI}=1.21$ to 2.08 for all-cause and $\mathrm{HR}=2.48$; 95\% CI 1.55 to 3.92 for cardiovascular mortality). Network score and marital status attenuated these associations by $27 \%(95 \% \mathrm{CI}$ $14 \%$ to $43 \%$ ) and $29 \%$ (95\% CI $17 \%$ to $52 \%$ ), respectively. In women, there was no consistent association between social support and mortality.

Conclusions The associations of social support with SEP and with mortality differ by gender. In men, social isolation and in particular not being married are important risk factors for mortality and are also likely to contribute to social inequalities in health.

\section{P2-292 TOTAL SERUM CHOLESTEROL AND CANCER INCIDENCE IN THE METABOLIC SYNDROME AND CANCER PROJECT (ME-CAN)}

doi:10.1136/jech.2011.142976k.25

${ }^{1} \mathrm{~S}$ Strohmaier, ${ }^{* 2,3} \mathrm{~T}^{\mathrm{T}}$ Bjørge, ${ }^{4} \mathrm{~J}$ Manjer, ${ }^{5} \mathrm{H}$ Concin, ${ }^{6} \mathrm{G}$ Nagel, ${ }^{7} \mathrm{~T}$ Stocks, ${ }^{7} \mathrm{P}$ Stattin ${ }^{1} \mathrm{H}$ Ulmer. ${ }^{1}$ Department of Medical Statistics, Informatics and Health Economics, Innsbruck, Austria; ${ }^{2}$ Department of Public Health and Primary Health Care, Bergen, Norway; ${ }^{3}$ Norwegian Institute of Public Health, Oslo, Norway; ${ }^{4}$ Department of Surgery, Malmö, Sweden; ${ }^{5}$ Agency for Preventive and Social Medicine, Bregenz, Austria; ${ }^{6}$ Institute of Epidemiology, Ulm, Germany; ${ }^{7}$ Department of Surgical and Perioperative Sciences, Umeå, Sweden

Introduction The relationship between serum cholesterol and cancer risk remains controversial. We examined the association between total serum cholesterol and cancer incidence in the Metabolic Syndrome and Cancer Project (Me-Can).

Methods Me-Can consists of seven cohorts from Norway, Austria, and Sweden including 289273 male and 288057 female participants prospectively followed up for cancer incidence $(n=39004)$ for a mean follow-up of 11.7 years. We used Cox regression models with age as the underlying time metric to calculate HRs and their $95 \%$ CIs for $1 \mathrm{mmol} / \mathrm{l}$ increment of total cholesterol levels adjusting for age at first measurement, body mass index and smoking. Additionally, we performed lag time analyses and corrected HRs for regression dilution bias.

Results Significant relationships of cholesterol with cancer incidence were all inverse including liver cancer ( $\mathrm{HR}=0.62$; 95\% CI 0.42 to $0.90)$ in males and cancers of the liver $(0.62 ; 0.49$ to 0.78$)$, gallbladder ( $0.62 ; 0.44$ to 0.85$)$, breast $(0.90 ; 0.85$ to 0.94$)$, and cancers of the lymph and haematopoietic tissue $(0.85 ; 0.76$ to 0.93$)$ in females. In lag analyses excluding cancer events occurring up to 5 years after cholesterol measurements, relationships persisted for liver, gallbladder and breast cancer.

Conclusions Total cholesterol was negatively associated with cancer risk at several sites. Lag time analyses suggested that these associations are only partially explained by reverse causation. 